\title{
Responsabilidad social universitaria: el papel de los medios de comunicación y sus stakeholders
}

\section{University social responsibility: the role of the media and its stakeholders}

Dra. Nereida Cea Esteruelas es profesora e investigadora de la Universidad de Málaga (España) (nereidacea@gmail.com) (https://orcid.org/0000-0002-7733-9366)

\begin{abstract}
Resumen
En este artículo se analiza la importancia creciente de la Responsabilidad Social Universitaria (RSU), impulsada por un cambio de visión por parte de los equipos rectores de las instituciones académicas. Ese cambio se materializa en diversos aspectos. En este trabajo nos centraremos en cómo la Universidad implementa sus principios de RSU mediante la comunicación con sus grupos de interés. Tras una revisión bibliográfica, se toma como estudio de caso la Universidad de Málaga y se analizan distintas acciones comunicativas encaminadas a que esta institución transmita a los distintos grupos de interés los aspectos referentes a su RSU. El análisis versa sobre dos escenarios comunicativos diferenciados: la comunicación directa de la misma Universidad a través de sus páginas corporativas y la comunicación de éstas a través de los medios de comunicación. Ambos tipos de acciones comunicativas tienen dimensiones diferenciadas, se dirigen a grupos de interés heterogéneos y cumplen misiones diversas. Por lo tanto, se tratan de manifestaciones distintas de un mismo objetivo, presidido en ambos casos por el principio de coherencia y correspondencia entre ambas. El análisis de la información institucional de la Universidad de Málaga muestra la importancia que ésta concede a la comunicación para transmitir a sus grupos de interés, además del grado de adecuación o cumplimiento con los principios de la responsabilidad social, su visión e identidad como organización.
\end{abstract}

\begin{abstract}
This article analyzes the growing importance of University Social Responsibility (USR), driven by a change of vision on the part of the governing teams of academic institutions. This change materializes in various aspects. This paper focuses on how the University implements its principles of USR through communication with its stakeholders. After a bibliographic review, Universidad de Málaga is taken as a case study and different communicative actions are analyzed related to how this institution informs referring to its USR to different interest groups. The analysis deals with two different communicative scenarios: direct communication of the University via its corporate pages, and the communication of its efforts through the media. Both types of communicative actions have differentiated dimensions and are directed to heterogeneous interest groups and work on different missions. Therefore, they are different manifestations of the same objective, guided in both cases by the principle of coherence and correspondence between the two. The analysis of the institutional information of Universidad de Málaga shows the importance of this responsibility, in addition to the degree of adequacy or compliance with the principles of social responsibility, their vision and identity as an organization.
\end{abstract}

\section{Palabras clave I keywords}

Responsabilidad social, responsabilidad social universitaria, medios de comunicación, comunicación institucional, comunicación corporativa.

Social responsibility, university social responsibility, media, institutional communication, corporate communication.

Cómo citar: Cea Esteruelas, N. (2018). Responsabilidad social universitaria: el papel de los medios de comunicación y sus stakeholders. Retos Revista de Ciencias de la Administración y Economía, 8(16), 95-105. https://doi.org/10.17163/ret.n16.2018.07 


\section{Introducción}

La preocupación de la sociedad y en particular de los agentes sociales sobre la Responsabilidad Social (en adelante, RS) es creciente. La Universidad, como agente socializador, también muestra su interés en desarrollar un modelo de responsabilidad social adaptado a su cometido y a su función social. En este sentido, a través de la Responsabilidad Social Universitaria (en adelante, RSU), las universidades adaptan y promueven estos principios a la esencia y naturaleza de su función como instituciones.

Durante la última década, la responsabilidad social en el ámbito empresarial, y posteriormente también en instituciones como la Universidad, ha ido cobrando importancia. Tanto en el ámbito empresarial, como en el de las instituciones políticas, los escándalos de diverso tipo han generado una mayor sensibilidad ante estos temas, lo que ha motivado por parte de empresas e instituciones una mayor necesidad de transparencia de sus actividades, un mayor compromiso y diálogo con la sociedad y un esfuerzo por parte de la organización de comunicar la aportación de ésta en términos de RS a sus grupos de interés.

En ese contexto de mayor concienciación de la repercusión que todo acto empresarial tiene en los mercados, en última instancia en las personas que los conforman y en la repercusión que tiene en la ciudadanía, cobra relevancia el concepto de responsabilidad social, esto es, responsabilidad social empresarial. Este paradigma ha tenido progresivamente su traslación a otras instituciones, bajo su adaptación a la naturaleza, misión y visión del organismo o institución. Este ha sido el caso de las universidades. Así pues, las universidades también han implementado los principios de responsabilidad social corporativa aplicados al contexto de su función social.

En este artículo se analiza la importancia creciente de la RSU, impulsada por un cambio de visión por parte de los equipos rectores de las instituciones académicas. Ese cambio se materializa, tal y como se detallará a continuación, en diversos aspectos. $\mathrm{Al}$ respecto, en este trabajo en concreto se describe cómo la Universidad implementa sus principios de RSU mediante la comunicación con sus grupos de interés. Para ello, el análisis versa sobre dos escenarios comunicativos diferenciados: la comunicación directa de la misma Universidad a través de sus páginas corporativas y la comunicación de éstas a través de los medios de comunicación. Ambos tipos de acciones comunicativas tienen dimensiones distintas, se dirigen a públicos en parte diferenciados y cumplen misiones distintas. Sin embargo, se tratan de manifestaciones distintas de un mismo objetivo por lo que, entre ambas, debe de presidir el principio de coherencia.

\section{Responsabilidad Social de la Universidad}

La RSU se encuentra en el punto de intersección entre: lo que la universidad quiere ser (su misión, visión y valores); lo que la universidad cree ser (la imagen que sus miembros tienen de ella); lo que la universidad es y hace (sus acciones y resultados) y lo que la sociedad espera de ella (las expectativas y opiniones de sus públicos externos) (Vallaeys, De la Cruz \& Siasa, 2009).

López \& Gil (2011) realizan una revisión bibliográfica sobre el concepto RSU y concluyen que existen dos formas de dar respuesta a esta cuestión. De una parte, se puede considerar que la Universidad, por su misma naturaleza, cumple con su función 
social, entendiendo por tal que esa misma responsabilidad social forma parte de su misión. Por otra parte, se puede añadir que las empresas e instituciones deben de cumplir y dar cuenta de su actuación responsable ante sus públicos interesados y, por ello, la Universidad, aunque tenga en su misma esencia esa función social, también debe cumplir con ese requisito de transparencia y diálogo con los agentes, públicos o grupos de interés que, en el caso que nos ocupa, bien se puede afirmar que por su complejidad y extensión es la misma sociedad civil.

Tomando como primer punto de análisis esa función social, y su proyección en la RSU, algunos ejemplos de misiones de RSU podrían ser los siguientes: establecimiento de comunidades dentro y fuera de la Universidad que promuevan responsabilidades sociales para las diferentes partes interesadas; promoción de actividades que sean éticas, inclusivas y beneficiosas para el público; énfasis en la conservación del medio ambiente, la sostenibilidad y el desarrollo social equilibrado; promoción del bienestar y la calidad de vida de las personas, especialmente de las poblaciones necesitadas y vulnerables; y el compromiso de construir un mundo mejor. Por lo general, estas misiones se logran a través de la enseñanza, la investigación y los servicios dentro de la comunidad universitaria y en colaboración con otros organismos.

En este mismo sentido, Ayala define la RSU como «la gerencia ética e inteligente de los impactos que genera la organización en su entorno humano, social y natural» (2011, p. 37). De acuerdo a esta forma de entender la responsabilidad social y el papel de la Universidad en la sociedad, ahondado en este concepto coincidimos en definir la RSU como:

La capacidad que tiene la Universidad de difundir y poner en práctica un conjunto de principios y valores generales y específicos, por medio de cuatro procesos claves: gestión, docencia, investigación y extensión (Domínguez, 2009, p. 39).

Vallaeys, De la Cruz \& Siasa (2009) han realizado uno de los mayores esfuerzos por definir y estudiar la responsabilidad social de la Universidad. En su libro Responsabilidad social universitaria proponen pensar la Universidad desde la clave de su responsabilidad social. Esto implica que este organismo tiene que «rendir cuentas de los impactos de su misión de formación, investigación y participación en la sociedad» (2009, p. 1). Para esta institución, los grupos de interés, por tanto, pasan a quedar englobados en un contexto más amplio como es la sociedad civil en su conjunto. En esa misma línea de entender la RSU como un mecanismo de control, Vallaeys (2014) habla del potencial de la RSU, ya que fuerza a la autocrítica institucional.

De acuerdo a este modelo teórico, la RSU supone ventajas y beneficios para la institución en los siguientes aspectos: pertinencia y permeabilidad social; coherencia e inte-gración institucional; dinámica institucional hacia la innovación; y racionalización de la gestión universitaria (Vallaeys, De la Cruz \& Siasa, 2009, p. 17).

La innovación juega un papel crucial para Leyva \& Farfán (2016), quienes considera que:

La proyección de la innovación educativa en el contexto de la responsabilidad social universitaria forma parte del itinerario cultural de las universidades y de las funciones fundamentales que sostienen su imaginario y praxis (p. 18). 
La RSU, aunque surge como algo distinto y tiene una finalidad específica, bien puede decirse que viene a funcionar como una plasmación de la misión y los valores de la institución universitaria. De ahí la importancia de que exista una correspondencia entre los principios rectores y cómo esos fundamentos se plasman y articulan con coherencia en la RSU. Se subraya así la importancia de la coherencia institucional que, como definen Vallaeys, De la Cruz \& Siasa, «significa a la vez coincidencia entre la acción y el discurso institucional y consistencia entre todas las áreas de la universidad» $(2009$, p. 2).

Es por ello que la RSU debería ocupar un lugar entre los mismos principios rectores de la Universidad, solo de esa forma tendría el máximo respaldo por parte de las autoridades académicas. Dichos principios de RSU formarían parte de:

La misión, visión, valores y/o proyecto educativo de la institución, así como su integración al proceso de planificación estratégica de la universidad con los recursos necesarios para la ejecución de los programas acordados (Vallaeys, De la Cruz \& Siasa, 2009, p. 25).

En ese mismo sentido, tal y como indica Domínguez (2009, p. 60), la Responsabilidad Social:

En su doble dimensión de exigencia ética y de estrategia racional de desarrollo para la inteligencia organizacional, pide a las organizaciones que respondan de sus acciones y consecuencias, al mundo y a los diversos grupos sociales afectados.

De ahí que la Universidad, dada su naturaleza como institución social con otros fines, se distancia del modelo de responsabilidad social empresarial, dando respuesta a estas premisas desde su posición como institución con una función social diferencial.

Sus obligaciones en el campo de la responsabilidad social pasan a responder a esa naturaleza diferencial. Efectivamente, de acuerdo a los objetivos de la Universidad, como agente de formación, tanto humana, como profesional, dentro del ámbito de los estrictamente académico, así como a su función como generadora de conocimientos a través de la investigación, también la Universidad intenta dar respuesta a la exigencia de cumplir con los principios de la responsabilidad social, pero siempre bajo ese modelo de institución.

Existe, por tanto, una correspondencia entre objetivos de la institución y su RSU. Es decir, como indica Aldeanueva y Jiménez (2013, p. 2) «cada universidad suele plasmar la Responsabilidad Social Universitaria en sus estrategias y tácticas de actuación».

Esa idea de pacto social de la Universidad es un concepto que propone Perić (2016), cuando afirma que, como instituciones responsables de la creación y difusión del conocimiento, las Universidades deben garantizar que este conocimiento esté en línea con las necesidades actuales y los requisitos futuros de la sociedad. Efectivamente, en un contexto de cambios económicos, políticos y sociales, se ha acometido una amplia reforma para enfrentar los nuevos desafíos sociales que estas instituciones enfrentan en la actualidad. La RSU también, desde su ámbito de acción, ha intentado dar respuesta a dimensión social de la Universidad y su importante papel en la sociedad ante los cambios acontecidos en las últimas décadas (Larrán \& Andrades, 2017).

También Vallaeys (2008) aborda el reto del pacto social de la Universidad y define esta cuestión de acuerdo al modo en el que se articula la preocupación de la 
Universidad por contribuir al desarrollo social. Es el entorno de una organización el que da sentido a la RS. "La responsabilidad social se desarrolla cuando una organización toma conciencia de sí misma, de su entorno, y de su papel en su entorno» (Vallaeys, 2008, p. 2).

En este sentido es importante subrayar cómo la RSU puede contribuir a la reputación y valor social de esta institución. Esa importancia de la cohesión social como principio ético y, en última instancia, razón social de la universidad también es un aspecto que subraya Beltrán, Bajos, \& Mata (2014).

Concretamente, la contribución social de la Universidad puede abordarse desde distintos enfoques, esto es, la Universidad puede intervenir en el desarrollo social mediante distintas acciones. Domínguez (2009, pp. 47-48), además de la contribución de la universidad en la sociedad mediante lo que denomina impactos educativos, cognoscitivos y epistemológicos, establece diferentes líneas de acción que articulan el impacto de la Universidad en su entorno y contribuyen al desarrollo social. Estos son: 1) la intervención de la Universidad de forma directa en su entorno más cercano, por ejemplo, mediante la creación de infraestructuras de atención a la población, tales como centros culturales, clínicas, etc. 2) intervención mediante actividades de impacto comunitario que, al igual que en las acciones anteriores tienden a cubrir necesidades específicas, las actividades se realizan en el entorno próximo, esto es, edificios públicos próximos al centro universitario; 3) actividades de asistencia técnica, mediante el apoyo a grupos que requieran de apoyo técnico-tecnológico o de transferencia de conocimiento para la mejora de sus resultados; y 4) actividades de voluntariado, en las que se fomenta que el alumnado se involucre en programas de voluntariado (Domínguez, 2009, pp. 47-48).

Ahondando en la importancia del entorno social y del grado de compromiso de la institución con éste, Vallaeys (2008) sugiere la necesidad de diseñar ese nuevo «contrato social» antes referido, entre la Universidad y la sociedad. Al respecto, se sugiere que cada Universidad y grupo docente diseñen su propia política de acción en relación con su propia identidad y contexto social (Vallaeys, 2008, p. 12).

Ese entorno social y el grado de compromiso de la universidad con la sociedad es la que, lo que permite que la responsabilidad social universitaria se haga realidad, esto es, "cuando la organización toma conciencia de sí misma, de su entorno y de su papel en dicho entorno» (Beltrán, Bajos, \& Mata, 2014).

Siguiendo a Vallaeys (2008), los tres grandes ejes de ese contrato social universitario serían los siguientes: la responsabilidad social de la ciencia, la formación a la ciudadanía democrática y la formación para el desarrollo. Vila (2012) coincide en el papel estructural que juega la ciudadanía en la concepción de la RSU. Al respecto, sugiere establecer mecanismos de comunicación interna y participación reales de la ciudadanía en los procesos.

La misma existencia de una política de RSU explícita es una forma de contribuir al desarrollo de ese contrato social, porque mediante la elaboración y aplicación de la RSU las autoridades identifican y se comprometen con esos principios rectores declarados como intenciones y que adoptan la forma de misión de la universidad y propósitos u objetivos (compromisos). En conclusión, la existencia de esa declaración de principios de RSU permite que tanto la misma institución, como el resto de agentes 
o grupos de interés (estudiantes, profesores, administrativos, sociedad civil...) puedan cotejar el grado de adecuación y cumplimiento de los principios de RSU declarados por la institución. Y, a su vez, es una forma de que las partes implicadas en el cumplimiento de esos principios de RSU tengan presentes esos principios y rijan su actividad y desempeño profesional para la consecución de tales fines.

\section{La RSU en la Universidad de Málaga}

Aunque cada vez se observa una mayor preocupación por parte de las Universidades por desarrollar políticas sociales, no hay que olvidar que su función es la formación académica y la contribución al avance del conocimiento. Su compromiso social se articula en torno a esos dos pilares. Y dando respuesta a esos dos pilares, se fomenta la inclusión social, la igualdad y otros aspectos relacionados con las libertades y los derechos humanos.

Shek \& Hollister (2017) realizan un análisis y revisión bibliográfica sobre la RSU, que complementan con estudios de caso aplicados en diferentes universidades americanas. En su análisis concluyen que las universidades estudiadas tienen diferentes objetivos y estrategias en lo que respecta a sus iniciativas de RSU; en segundo lugar, observaron que existen distintos plantes de RSU, de acuerdo a niveles de recursos y compromisos, que pueden proporcionar excelentes marcos de referencia para el desarrollo de las políticas y programas de la RSU de otras instituciones. En línea con esto, sugieren que también es necesario intensificar el trabajo de evaluación de las iniciativas de RSU, mediante trabajos de evaluación de los programas e iniciativas de RSU, particularmente aquellas que analizan el impacto de la RSU en los stakeholders.

En España, González et al. (2016) aplican una metodología similar de análisis de aplicación de la RSU y concluyen que menos de la mitad de las universidades del Sistema Universitario Español tienen un plan de Responsabilidad Social Universitaria.

La implantación de la RSU en España surge en el contexto de modernización de la Universidad en España y tiene como telón de fondo el proceso de modernización de esta institución en toda Europa, siguiendo las recomendaciones de la Comisión Europea.

Con esos antecedentes, en nuestro país se elabora el plan Estrategia Universidad 2015, como iniciativa coordinada entre el Gobierno de España, las Comunidades Autónomas y las Universidades, «mediante la promoción de la excelencia en formación e investigación, la internacionalización del sistema universitario y su implicación en el cambio económico basado en el conocimiento y en la mejora de la innovación» (Ministerio de Educación, 2009). Dicho Plan articula esa necesidad de implantación progresiva en las universidades de planes de RS.

De las once universidades andaluzas, todas ellas, exceptuando una, realizan acciones de RSU y, además, salvo dos, el resto forman parte del Foro de los Consejos Sociales de las Universidades andaluzas, que funciona como una red o grupo de trabajo en el ámbito de la Responsabilidad Social.

Una de esas universidades comprometidas en el desarrollo de un plan de acción de RSU es la Universidad de Málaga (UMA) que, en la línea de sus homólogas, explicita su compromiso con la sociedad y con sus grupos de interés a través del Plan de 
RSU. Esta, como otras instituciones docentes, fomenta un diálogo con su comunidad y estimula la relación de intercambio con la sociedad, tal y como queda reflejado en su Memoria de RSU.

\section{El papel de la comunicación en la articulación de la RSU}

A través de la información institucional, la Universidad transmite su visión, el modelo que define su identidad como organización y la cultura organizativa que rige en ella. La comunicación con los grupos de interés es un proceso continuo, constante y fluido. Asimismo, debería estar presidido por un espíritu dialógico. Nos referimos por tanto a un proceso comunicativo y dialógico con sus stakeholder, más allá de la rendición anual de cuentas, que es un acto comunicativo en el que se compendia toda la actividad realizada durante el curso académico.

El hecho de que la Universidad se mantenga en actitud atenta a las demandas de los grupos de interés, no significa que deba mantener un diálogo constante, pero sí habilitar los mecanismos que hagan posible que sea permeable al intercambio de información y opinión sobre cómo mejorar y cumplir de esa forma mejor con su misión y objetivos.

Partiendo de la premisa de que las universidades tienen distintos grupos de interés, se analiza a continuación las características de la comunicación con cada uno de estos públicos. Siguiendo la propuesta de clasificación de Gaete (2011, p. 116), pueden considerarse stakeholders, en primer lugar, los estudiantes, asimismo, las empresas y otros organismos sociales; también se incluye al Gobierno, así como a los proveedores de recursos para el funcionamiento de la institución; y, por último, aunque no por ello menos importante todo el personal que desempeña su trabajo dentro de la institución.

En primer lugar, cabe destacar la labor que anualmente realiza el equipo Rector de la Universidad de Málaga mediante el informe o memoria de progreso referente a los ámbitos de aplicación de la RS, siguiendo las recomendaciones del anteriormente referido Plan Estrategia Universidad 2015. En el caso que nos ocupa, el informe de RSU de la Universidad de Málaga se articula en torno a ocho bloques. En dicho informe se da cuenta de las actuaciones en cada uno de los ámbitos que engloba la RSU. Cada bloque agrupa el compromiso de la Universidad de Málaga con los siguientes aspectos: 1) la organización y recursos; 2) el alumnado; 3) el personal; 4) la sociedad; 5) el medio ambiente; 6) la investigación y la transferencia de conocimiento al sector empresarial; 7) la mejora constante y la transparencia; 8) la internacionalización (Universidad de Málaga, 2016, p. 5).

Sin embargo, tal y como indica en su Memoria la Universidad de Málaga, el principal grupo de interés son sus estudiantes. Así se recoge también en sus Estatutos, en los que se subraya la misión y la visión de esta institución académica.

La memoria anual de RSU de la Universidad de Málaga se viene elaborando desde hace años y supone, por una parte, un esfuerzo de compendio de revisión y cotejo de las acciones realizadas por esta institución en materia de responsabilidad social; además, contribuye a la comunicación con los agentes implicados, al darles cuenta del desarrollo de las distintas actividades y planes estratégicos encaminados a implementar el compromiso de la Universidad de Málaga con la RSU. 
Cabe destacar que las referidas Memorias, además de servir como herramienta de transparencia de las actuaciones de esta institución, también permiten una autoevaluación para la misma institución. De hecho, la misma memoria recoge en su apartado final la autocalificación, verificación y correspondencia de la misma con respecto a los principios y sugerencias establecidos en Global Reporting Initiative (G4). Asimismo, dicho documento incorpora las Recomendaciones del Foro de los Consejos Sociales de las Universidades Públicas de Andalucía y las directrices del Plan Estratégico de la Universidad de Málaga.

Uno de los aspectos en los que la Memoria hace hincapié es en la dimensión social de su compromiso con los grupos de interés, en concreto con la inclusión a través de su programa de intervención psicopedagógica. Esta institución fue la primera universidad pública que recibió su reconocimiento como institución socialmente responsable con la diversidad funcional, al ofrecer herramientas para integrar políticas de igualdad en todas sus áreas.

Por otra parte, cabe destacar que la Universidad de Málaga articula todas sus actuaciones englobadas dentro del paraguas de la RSU a través del Servicio de Calidad, Planificación Estratégica y Responsabilidad Social. La función del Servicio de Calidad es proporcionar apoyo técnico, asesoramiento y formación a todas las Unidades de la Universidad para difundir la cultura de la calidad y fomentar e implantar la mejora continua y la excelencia en todos los ámbitos de la comunidad universitaria.

El servicio de Calidad es, además, el encargado de la elaboración de la Memoria de RSU. Asimismo, mantiene una información constantemente actualizada con todos los grupos de interés. Este servicio atiende de forma global al grupo de interés formado por el alumnado y egresados, facilitando todo tipo de información que puede ser de interés para este grupo. Entre la información de resultados de interés para el alumnado, este servicio informa periódicamente sobre alumnos matriculados y procedencia, alumnos en movilidad internacional, tasa de éxito y rendimiento, la oferta y demanda de matriculación de títulos de grado y la evolución del número de alumnos egresados, todo ello mediante un análisis comparativo de las tasas cada año.

Asimismo, realiza diferentes informes de resultados sobre la inserción laboral y las prácticas en empresas del alumnado. Este aspecto es de gran interés porque en sus informes se analiza la tasa de inserción laboral comparando la situación del alumnado al año de haber terminado y posteriormente dos años después. Asimismo, esta información se completa con un análisis de empleabilidad de los alumnos egresados desde 2010, lo que permite analizar la evolución de forma periódica. A esto se suman informes anuales sobre el grado de desempeño en prácticas externas curriculares.

De gran interés, no solo para el grupo del estudiantado, sino para toda la comunidad académica, son los informes comparativos sobre las encuestas de opinión del alumnado sobre la actuación docente del profesorado, las encuestas de clima laboral del personal, las encuestas de satisfacción de los alumnos respecto a la organización de los títulos y centros y la encuesta de satisfacción de los egresados, todas ellas con carácter anual y que incluyen un análisis evolutivo de los resultados obtenidos desde su implantación.

Asimismo, existe un servicio de comunicación centralizado que permite articular distintas vías de comunicación de la Universidad con los distintos grupos de interés. 
Además de la información directa del Servicio de Calidad a través de la página web de la institución, el Servicio de Comunicación se encarga de las relaciones con los medios de comunicación, la comunicación interna, la edición y mantenimiento de la web institucional y todo tipo de acciones comunicativas a través de las redes sociales.

Las distintas acciones de comunicación antes mencionadas cubren, por tanto, la totalidad de los grupos de interés. Mientras que el personal no docente y docente-investigador tiene canales propios de comunicación, restringidos a estos grupos, tales como la página web y la comunicación a través de email y grupos de distribución, los estudiantes y egresados, además de la información disponible en la misma página web, disponen de las noticias que publican los medios de comunicación tanto a través del servicio de comunicación de la Universidad de Málaga, lo que incluye la radio Comutopia (emisora de la radio y televisión universitaria), distintos espacios fijos en los medios públicos y privados de ámbito regional y la cobertura que cada medio da a la información que proporciona el gabinete de comunicación de la Universidad de Málaga.

\section{Discusión y conclusiones}

La revisión de distintos autores, nos permite aportar algunas conclusiones y sugerencias de mejora ante el proceso de rendición de cuentas de las universidades ante sus grupos de interés o stakeholders. Dado el carácter público y su función social, como señala Gaete (2011) tienen la obligación de explicar a la sociedad el grado de cumplimiento de su función social.

En este trabajo se analiza la Universidad de Málaga y las distintas acciones comunicativas encaminadas a que esta institución transmita a los distintos grupos de interés los aspectos referentes a su RSU.

Tal y como considera Larrán y López (2010), sería recomendable que esta acción se completara con un sistema de verificación externa, del tipo Global Reporting Iniciative, lo que permitiría una autoevaluación de la validez de estas memorias. El sistema promovido por este organismo permite la autoevaluación interna y también externa para muchas instituciones. Este organismo, mediante un acuerdo internacional, promueve las pautas para orientar a las empresas e instituciones en la elaboración de sus memorias. Asimismo, toda empresa u organismo puede solicitar evaluar su memoria para conocer el grado de validez de la misma, tal y como ya hace la Universidad de Málaga. A su vez, esto permite realizar estudios sobre el grado de adaptación de las distintas instituciones y centros a las recomendaciones realizadas por este organismo. Este es el caso del análisis realizado por Huerta-Riveros y Gaete-Feres (2017) en Latinoamérica y Caribe, en el que los autores referidos concluyen que las Universidades que aplican el sistema de verificación Global Reporting Iniciative representan el 17\% del total de universidades.

Por otra parte, como señala Gaete (2011), la elaboración de memorias de RSU, dado que tiene su fundamentación en un proceso de accountability, la elaboración y transparencia de los indicadores seguidos permiten a los agentes implicados evaluar el impacto de las estrategias y acciones implementadas por la institución en el campo de la RSU.

El análisis de la información institucional de la Universidad muestra la importancia que ésta concede a la comunicación para transmitir a sus grupos de interés, ade- 
más del grado de adecuación o cumplimiento con los principios de la responsabilidad social, su visión e identidad como organización.

Más allá de la memoria anual, esta institución establece un flujo constante de comunicación con sus grupos de interés de forma constante a través de su página web. Las noticias relacionadas con todo tipo de iniciativas ocupan una parte importante de la home page de su página web. Por tanto, desde el departamento encargado de la comunicación se considera que la comunicación con los grupos de interés debe ser un proceso fluido que va más allá del proceso de rendición de cuentas anual mediante la memoria de RSU.

Entre los mecanismos que hacen posible ese intercambio de información y opinión con los grupos de interés está la oficina de atención a los estudiantes y un espacio en la web donde se atiende todo tipo de sugerencias y peticiones de información.

Dentro del conjunto de los stakeholders, la Universidad concede prioridad al alumnado. Así se recoge también en sus Estatutos, en los que se subraya la misión y la visión de esta institución académica. Para coordinar la comunicación con este grupo de interés, la institución tiene habilitadas diferentes vías, tal y como se ha referido. Destacamos la importancia que se concede al proceso de feedback y, por ende, la Universidad mantienen constantes encuestas de autoevaluación de su labor y atención al alumnado.

Asimismo, también la Universidad atiende las demandas de información del resto de grupos de interés, tales como empresas y otros organismos sociales, Gobierno, proveedores y todo el personal que desempeña su trabajo dentro de la institución. Nuevamente, la página institucional viene funcionando como canal de comunicación constante y fluido también para estos grupos de interés.

Igualmente, a modo de compendio, en la Memoria anual, el equipo rector da cuenta del progreso de la institución en los distintos ámbitos de aplicación de la RS. La estructura que mantiene la Memoria cubre los aspectos más significativos de la RS, tales como la organización y recursos; las actividades propias de la institución, esto es la investigación y la transferencia de conocimiento al sector empresarial, el grupo de personal, alumnado y, por extensión la sociedad; su preocupación por el medio ambiente, la mejora constante y la transparencia; y, por último, su grado de compromiso con la internacionalización de la institución.

En definitiva, la institución analizada muestra la importancia del cumplimiento con los principios de la RSU, aspecto éste del que se da cuenta anualmente mediante la elaboración de una Memoria. Siendo este aspecto de crucial importancia para que los distintos grupos de interés evalúen el grado de adecuación y cumplimiento de la dimensión social de la actividad de esta institución, es importante reforzar los aspectos comunicativos mediante un flujo constante de información con los grupos de interés. Mediante el departamento de comunicación y la labor coordinada de éste con el departamento de Calidad de esta Universidad, se logra mantener ese flujo comunicativo constante con los distintos grupos de interés, que redunda en mejorar la imagen social de esta institución.

En conclusión, si coincidimos en que:

La Universidad tiene la responsabilidad social de promover el debate, facilitarlo, conducirlo y enriquecerlo, dando al público ciudadano los medios para informarse, reflexionar y juzgar y a las empresas los conocimientos adecuados para aplicar su propia responsabilidad social (Vallaeys, 2008, p. 14). 
Serán necesarios seguir creando mecanismos de difusión del grado de seguimiento de esos principios, lo cual redundará en una mejora de la imagen de esta institución y de la percepción del valor social de la misma por parte de la sociedad civil.

\section{Referencias}

Aldeanueva, I., \& Jiménez, J. A. (2013). Experiencias internacionales en materia de responsabilidad social universitaria. Visión de futuro, 17(1), 1-16.

Ayala, M. (2011) Responsabilidad social universitaria. Realidad y reflexión, (33), 29-38.

Beltrán, J., Bajos, E., \& Mata, A. (2014). La responsabilidad social universitaria, el reto de su construcción permanente. Revista Iberoamericana de Educación Superior, 5(14), 3-18.

Domínguez, M. J. (2009). Responsabilidad social universitaria. Humanismo y trabajo social, 8, 36-67.

Gaete, R. (2011). La responsabilidad social universitaria como desafío para la gestión estratégica de la Educación Superior: el caso de España. Revista de Educación, 355, 109-133.

Global Reporting Initiative- GRI (2013). Guía para la elaboración de memorias de sostenibilidad. Principios y contenidos básicos. Ámsterdam (Países Bajos): Global Reporting Initiative.

González, O., Fontaneda, I., Camino, M. A. \& Revilla, A. (2016). La Responsabilidad Social en las Universidades Españolas 2014/15. Essays and Researchs, (12), s/p.

Huerta-Riveros, P., \& Gaete-Feres, H. (2017). Responsabilidad social universitaria a través de los reportes de sostenibilidad del Global Reporting Initiative: experiencia de una universidad pública. Revista Iberoamericana de Educación Superior, 8(23), 120-137.

Larrán, J., \& Andrades, F. J. (2015). Análisis de la responsabilidad social universitaria desde diferentes enfoques teóricos. Revista Iberoamericana de Educación Superior, 6(15), 91-107. https:// doi.org/10.1016/S2007-2872(15)30005-6

Larrán, M. \& López, A. (2010) Una propuesta de memoria de sostenibilidad universitaria como vía de diálogo con los diferentes grupos de interés. En M. De la Cuesta, C. De la Cruz \& J. M. Rodríguez (Coords.), Responsabilidad social universitaria. La Coruña: Netbiblo.

Leyva, A., \& Farfán, P. C. (2016). La innovación educativa en el ámbito de la responsabilidad social universitaria. Revista Cubana de Educación Superior, 35(2), 16-34.

López, S. \& Gil, A. (2011). Responsabilidad social universitaria. Líneas para el Debate, 44, s.p.

Ministerio de Educación (2009). Estrategia Universidad 2015. Madrid: Ministerio de Educación.

Rodríguez, J.M. (2010). Responsabilidad social universitaria: del discurso simbólico a los desafíos reales. En M. De la Cuesta, C. De la Cruz \& J. M. Rodríguez (Coords.), Responsabilidad Social Universitaria. La Coruña: Netbiblo.

Shek, D. \& Hollister, R. (2017). University Social Responsibility and Quality of Life. Singapore: Springer.

Vallaeys, F. (2008). ¿Qué es la responsabilidad social universitaria. (https://goo.gl/WyjfUU).

Vallaeys, F. (2014). La responsabilidad social universitaria: un nuevo modelo universitario contra la mercantilización. Revista Iberoamericana de Educación Superior, 5(12), 105-117. https:// doi.org/10.1016/S2007-2872(14)71945-6

Vallaeys, F., De la Cruz, C \& Sasia, P. (2009). Responsabilidad social universitaria. Manual de primeros pasos. México, D.F.: McGraw-Hill Interamericana editores.

Vila, E. S. (2012). Ciudadanía, equidad e innovación: reflexiones sobre la política de responsabilidad social de las universidades. Innovación Educativa, 12(59), 61-85. 